\title{
Simple classical approach to spin resonance phenomena
}

\section{Gordon, R A}

\section{Published in:}

American Journal of Physics

Link to article, DOI:

10.1119/1.11021

Publication date:

1977

\section{Document Version}

Publisher's PDF, also known as Version of record

Link back to DTU Orbit

Citation (APA):

Gordon, R. A. (1977). Simple classical approach to spin resonance phenomena. American Journal of Physics, 45(6), 563-568. https://doi.org/10.1119/1.11021

\section{General rights}

Copyright and moral rights for the publications made accessible in the public portal are retained by the authors and/or other copyright owners and it is a condition of accessing publications that users recognise and abide by the legal requirements associated with these rights.

- Users may download and print one copy of any publication from the public portal for the purpose of private study or research.

- You may not further distribute the material or use it for any profit-making activity or commercial gain

- You may freely distribute the URL identifying the publication in the public portal

If you believe that this document breaches copyright please contact us providing details, and we will remove access to the work immediately and investigate your claim. 


\title{
Simple classical approach to spin resonance phenomena
}

\author{
R. A. Gordon \\ Physics Laboratory I, The Technical University of Denmark, DK-2800 Lyngby, Denmark \\ (Received 7 June 1976; revised 29 December 1976)
}

\begin{abstract}
A simple classical method of describing spin resonance in terms of the average power absorbed by a spin system is discussed. The method has several advantages over more conventional treatments, and a number of important spin resonance phenomena, not normally considered at the introductory level, are calculated in simple quantitative fashion.
\end{abstract}

\section{INTRODUCTION}

Classical introductions to spin resonance are generally based on calculations of the magnetization of a spin system using the Bloch equations. ${ }^{1-4}$ While such an approach often provides a convenient classical description of many features of spin resonance, it suffers from the disadvantage that the Bloch equations are often difficult to solve and frequently invalid for even the simplest forms of spin resonance, such as EPR or NMR. ${ }^{5}$ In addition, many important spin resonance phenomena such as nuclear quadrupole resonance (NQR), nuclear acoustic resonance (NAR), or various forms of coupled or double-spin resonance are generally ignored completely or treated only in qualitative fashion at the introductory level. Such spin resonance phenomena can, of course, be treated quantitatively using quantum-mechanical perturbation theory, ${ }^{5-10}$ but such techniques are generally too advanced for students encountering spin resonance for the first time and do not, in any case, provide the ready physical insight characteristic of classical methods.

In this paper, we discuss an alternative method of presenting spin resonance that has several advantages over the more conventional approaches mentioned above. The technique is to calculate classically the average puwer absorbed by a spin system. As will be shown below, such a calculation has the important advantage that the resonant spin response can be found from the free undamped motion of the spin system, i.e., without the introduction of the Bloch equations. The method thus possesses the generality of the quantum-mechanical approach while retaining and even enhancing the simplicity and appeal of classical methods. The method is first illustrated for the simple standard form of spin resonance and then applied to a number of other important spin resonance phenomena which, to our knowledge, have not been treated in a simple quantitative manner at the introductory level before.

\section{AVERAGE POWER} as

The average power absorbed by a spin system is defined

$$
\left\langle\frac{\partial \epsilon}{\partial t}\right\rangle \equiv \lim _{T \rightarrow \infty}\left(\frac{1}{T}\right) \int_{0}^{T}\left(\frac{\partial \epsilon}{\partial t}\right) d t
$$

where $\epsilon$ is the energy of the spin system and $T$ is the time over which the averaging takes place. The averaging process could be taken over shorter finite time intervals, thus allowing a number of transient phenomena to be calculated ${ }^{11}$ but, for simplicity, we consider only the case $T \rightarrow \infty$, corresponding to the usual steady-state conditions assumed in introductory treatments. The definition of the spin response in terms of Eq. (1) leads naturally to the phenomenon of spin resonance since $\langle\partial \epsilon / \partial t\rangle$ will be appreciable only when the frequency of the small time-varying electromagnetic field used to excite the resonance is close to one of the spin resonance frequencies. In most cases, this resonant behavior will be well described by the dominant first-order term ${ }^{12,13}$ of the power series expansion of $\partial \epsilon / \partial t$ in this small timevarying electromagnetic field. The advantage of the average-power calculation follows from the fact that this firstorder term of $\partial \epsilon / \partial t$ can be calculated from the free undamped response of the spin system, i.e., without explicitly considering the driving field or introducing spin relaxation effects. ${ }^{14}$ This not only leads to a significant simplification of the calculations but also means that the results obtained will not be restricted by the validity of particular spin-relaxation models, e.g., the phenomenological Bloch equations. Thus the first-order, average-power calculation is particularly well suited to describing a wide range of spin resonance phenomena in a simple unified manner. Below, we use the simple $\simeq$ to emphasize that only this first-order term is retained. We now illustrate such a calculation for the usual form of spin resonance treated in elementary texts.

\section{MAGNETIC SPIN RESONANCE (e.g., NMR, EPR)}

A fundamental property of many physical systems is the existence of an internal spin degree of freedom characterized by the presence of a nonzero spin magnetization vector. In the classical limit, this spin magnetization vector will precess about specific directions defined by an external electromagnetic field. ${ }^{15}$ An important example of such a precession is the so-called magnetic-dipole spin precession, ${ }^{16}$ in which the spin magnetization vector precesses about an external magnetic field. In practice, this magnetic-dipole spin precession is often easiest to observe using the technique of magnetic spin resonance. ${ }^{17}$

In magnetic spin resonance ${ }^{1-7}$ a small ac magnetic field is used to drive the magnetic-dipole precession of a spin system resonantly in the presence of a much larger dc magnetic field. The energy, $\epsilon$, and the precession of the spin system are given classically as ${ }^{18}$

$$
\begin{gathered}
\epsilon=-\left(M_{S} B\right)(\hat{S} \cdot \hat{B}), \\
d S / \partial t=\left(M_{S} B\right)(\hat{S} \times \hat{B}) \equiv \omega_{B} \times S,
\end{gathered}
$$

where $\omega_{B}$ is the angular frequency with which the spin magnetization vector, $\mathbf{S}$, precesses about the total magnetic ficld B. Here $M_{S}$ is the magnetic dipole moment of the spin 


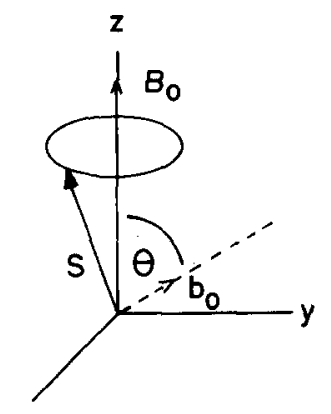

$X$ a. MAGNETIC SPIN RESONANCE

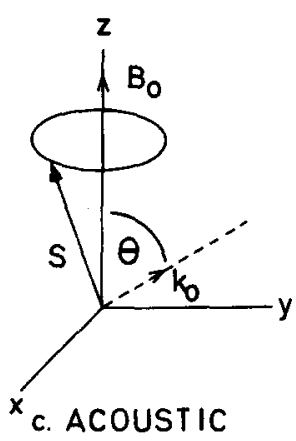

SPIN RESONANCE

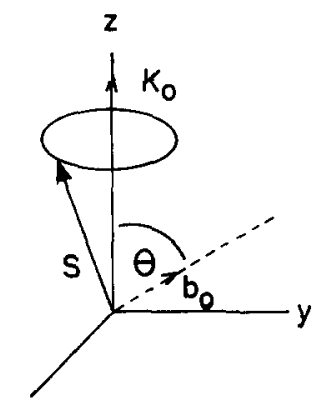
SPIN RESONANCE

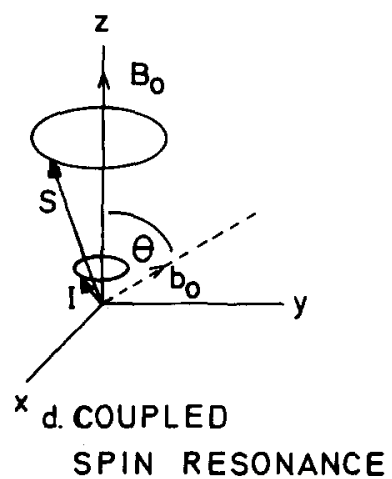

$x$ b. QUADRUPOLE

Fig. 1. $\mathbf{B}_{0}$ and $\mathbf{K}_{0}$, respectively, represent large dc magnetic fields and electric field gradients applied to spin systems with spin magnetization vectors given by $\mathbf{S}$ and $\mathbf{I}$. $\mathbf{b}_{0}$ and $\mathbf{k}_{0}$, respectively, represent small ac magnetic fields or electric field gradients in the $y-z$ plane used to drive various spin precessions into resonance as described in the text.

system. Without any loss of generality, the dc and ac magnetic fields can be taken as $\mathbf{B}_{0}$ and $\mathbf{b}=\mathbf{b}_{0} \sin (\omega t+\phi)$, respectively, as shown in Fig. 1(a). ${ }^{19}$ The power absorbed, $\partial \epsilon / \partial t$, follows from Eqs. (2) and (3) as ${ }^{20}$

$$
\partial \epsilon / \partial t=-\left(M_{S}\right)(\hat{S} \cdot d \mathbf{b} / d t) \text {. }
$$

The free undamped motion of $\mathbf{S}$ is found by neglecting the small driving field $\mathbf{b}$ in Eq. (3), i.e.,

$$
\begin{gathered}
S_{x} \simeq S_{t} \sin \left(\omega_{B} t\right), \quad S_{y} \simeq S_{t} \cos \left(\omega_{B} t\right), \\
S_{z}, S_{t} \simeq \mathrm{const}, \\
\omega_{B} \simeq\left(M_{S} / S\right) B_{0},
\end{gathered}
$$

where $S_{t}$ denotes the transverse component of $S$ given by $S^{2}=S_{t}{ }^{2}+S_{z}{ }^{2}$. Substituting Eqs. (4) and (5) into Eq. (1) for $\langle\partial \epsilon / \partial t\rangle$ yields

$$
\begin{aligned}
&\left\langle\frac{\partial \epsilon}{\partial t}\right\rangle_{\mathrm{MR}} \simeq-\left(\frac{M_{S}}{S}\right) b_{0} \omega \lim _{T \rightarrow \infty} \\
& \frac{1}{T} \int_{0}^{T}\left(S_{y} \sin \theta\right. \\
&\left.+S_{z} \cos \theta\right) \cos (\omega t+\phi) d t \\
& \simeq-\frac{1}{2}\left(\frac{M_{S}}{S}\right) S_{t} b_{0} \omega \sin \theta \cos \phi \delta_{\omega, \omega_{B}}
\end{aligned}
$$

where the Kronecker delta, $\delta_{\omega, \omega_{B}}$, shows explicitly the resonant nature of $\langle\partial \epsilon / \partial t\rangle .{ }^{11}$ The above integration demonstrates in direct fashion that the energy absorption occurs only from that component of the ac driving field which is perpendicular to $\mathbf{B}_{0}$ (viz. the factor $\sin \theta$ ), out of phase with $S_{y}$ (viz. the factor $\cos \phi$ ), and which has the same sense of circular polarization as the precessing spin [viz. only the $\cos \left(\omega-\omega_{B}\right) t$ term in the integrand of Eq. (6) contributes to $\langle\partial \epsilon / \partial t\rangle]$. Thus, it is unnecessary to artificially assume rotating or circularly polarized ac magnetic fields as is generally done in classical treatments. ${ }^{5}$ We now consider how the inclusion of electric quadrupole effects in Eqs. (2) and (3) makes possible other forms of spin resonance which can be calculated by the same method.

\section{QUADRUPOLE EFFECTS}

The energy and precession of a spin system which possesses both a magnetic dipole moment, $M_{S}$, and an electric quadrupole moment, $Q_{S},{ }^{21}$ will be given by

$$
\begin{array}{r}
\epsilon=-\left(M_{S} B\right)(\hat{S} \cdot \hat{B})-(1 / 2)\left(Q_{S} K\right)\left[3(\hat{S} \cdot \hat{K})^{2}-1\right], \\
d \mathbf{S} / d t=\left(M_{S} B\right)(\hat{S} \times \hat{B})+3\left(Q_{S} K\right)(\hat{S} \cdot \hat{K})(\hat{S} \times \hat{K}) \\
\equiv\left(\omega_{B}+\omega_{Q}\right) \times \mathbf{S},
\end{array}
$$

where $\mathbf{K}^{22}$ is the electric field gradient vector. ${ }^{23}$ Equations (8) and (9) show that $\mathbf{S}$ precesses simultaneously about the magnetic field $\mathbf{B}$ (at the frequency $\omega_{B}$ ) and the electric field gradient $\mathbf{K}$ (at the frequency $\omega_{Q}$ ). The latter precession is referred to as the electric quadrupole spin precession while the former precession is simply the magnetic-dipole spin precession discussed above. Since the general motion of the spin system consists of both precessions, it is possible to use either of the two precessions to drive the other precession into resonance. ${ }^{24}$ This leads to two complementary types of spin resonance that are each capable of providing unique information about the spin system and its environment. We now consider explicitly these two important types of spin resonance in a more quantitative fashion using the average-power method.

\section{QUADRUPOLE SPIN RESONANCE (e.g., NQR)}

In this form of spin resonance, ${ }^{1-3,8,17}$ a small linearly polarized ac magnetc field $\mathbf{b}=\mathbf{b}_{0} \sin (\omega t+\phi)$ is used to drive the electric quadrupole spin precession of a spin $\mathbf{S}$ about a large dc electric field gradient $\mathbf{K}_{0}$ as shown in Fig. 1(b).${ }^{19}$ Since the electric quadrupole spin precession will be a sensitive function of both $Q_{S}$ and $\mathbf{K}_{0}$, quadrupole spin resonance experiments will give important information about the electric charge distributions of both the spin system and its environment. A significant practical advantage in carrying out such experiments is the fact that the experimental techniques employed are often similar to those used in conventional magnetic spin resonance experiments where a small ac magnetic field is also used to excite the resonant spin response. Another advantage, in many cases, is that the electric field gradient, $\mathbf{K}_{\mathbf{0}}$, need not be applied externally since large dc electric field gradients often exist at many spin systems due to their particular environment. In fact, it is the value of $\mathbf{K}_{\mathbf{0}}$ itself which is usually of primary interest in such cases. We now consider how these features appear in the average-power method.

A brief calculation using Eqs. (8) and (9) shows that the expression for $\partial \epsilon / \partial t$ remains the same as in the case of magnetic spin resonance [Eq. (4)], ${ }^{20}$ while the free undamped precession of $\mathbf{S}$ is found from Eq. (9) by ignoring b; i.e.,

$$
\begin{gathered}
S_{x} \simeq S_{t} \sin \left(\omega_{Q} t\right), \quad S_{y} \simeq S_{t} \cos \left(\omega_{Q} t\right), \\
S_{z}, S_{t} \simeq \text { const } \\
\omega_{Q} \simeq 3\left(Q_{S} / S\right)\left(S_{z} / S\right) K_{0} .
\end{gathered}
$$


Substituting Eqs. (4) and (10) into Eq. (1) then yields ${ }^{11}$

$\langle\partial \epsilon / \partial t\rangle_{\mathrm{QR}}$

$$
\simeq-(1 / 2)\left(M_{S} / S\right) S_{1} b_{0} \omega \sin \theta \cos \phi \delta_{\omega, \omega Q} .
$$

showing that the energy is absorbed from the same perpendicular, out-of-phase, circularly polarized component of the ac magnetic field as was responsible for magnetic spin resonance. The factor $\delta_{\omega, \omega Q}$, however, leads to important differences between the phenomena, namely, the explicit dependence of $\langle\partial \epsilon / \partial t\rangle$ on the spin orientation (since $\omega_{Q}$ is proportional to $S_{z} / S$ ) and the requirement that the angular frequency, $\omega$, be varied in order to satisfy the resonance condition $\omega=\omega_{Q}$ (since $\omega_{Q}$ is also proportional to $K_{0}$ which, as was noted above, is generally fixed by the spin environment).

\section{ACOUSTIC SPIN RESONANCE (e.g., NAR, ACOUSTIC EPR)}

In contrast to the case of quadrupole spin resonance, the electric quadrupole precession is dominant, acoustic spin resonance corresponds to the opposite limiting case where the magnetic-dipole spin precession is dominant. Thus in acoustic spin resonance ${ }^{9.17}$ a small, linearly polarized ac electric field gradient $\mathbf{k}=\mathbf{k}_{0} \sin (\omega t+\phi)$ is used to drive the magnetic-dipole spin precession of a spin, $\mathbf{S}$, about a large dc magnetic field, $\mathbf{B}_{0}$, as shown in Fig. 1(c) ${ }^{19}$ The term acoustic spin resonance arises from the fact that the ac electric field gradient is usually generated using an acoustic wave to modulate the electric charge distribution in the vicinity of the spin system. This requires different and generally less sensitive, experimental techniques than those employed in magnetic spin resonance or quadrupole spin resonance, where the time-varying electromagnetic field used to excite the resonance is generated directly. On the other hand, unlike direct electromagnetic methods, acoustic excitation can be applied to electrically opaque materials such as metals and is also a more versatile means of excitation since both longitudinal and transverse acoustic waves can be employed. Thus, acoustic spin resonance will, in general, depend on both the orientation of the ac electric field gradient and the orientation of the wave vector of the acoustic wave used to generate this ac electric field gradient. This allows for a more complete specification of the electrical and magnetic properties of the spin system and its environment than is possible by purely electromagnetic methods. Here, for simplicity, we consider acoustic spin resonance directly in terms of the ac electric field gradient rather than in terms of the acoustic wave vector. In the case of a pure longitudinal acoustic wave this will, of course, not lead to any differences in the angular dependence of the phenomenon since the acoustic wave vector will then be parallel to the ac electric field gradient.

The power absorbed in acoustic spin resonance is found by a straightforward calculation of $\partial \epsilon / \partial t$ from Eqs. (8) and (9) ${ }^{25,26}$ :

$$
\frac{\partial \epsilon}{\partial t}=-\frac{1}{2} Q_{s}\left[3(\hat{S} \cdot \hat{k})^{2}-1\right] \frac{d k}{d t},
$$

where $k$ is the instantaneous amplitude of $\mathbf{k}$; i.e., $k=k_{0}$ $\sin (\omega t+\phi)$. The free undamped precession of $\mathbf{S}$ about $\mathbf{B}_{0}$ is again given by Eq. (5), so that ${ }^{11}$

$$
\begin{aligned}
\langle\partial t / \partial t\rangle_{\wedge \mathrm{R}} \simeq-(3 / 4)\left(Q_{S} S_{t} \omega k_{0} \cos \phi / S^{2}\right) \\
\times\left[S_{z} \sin (2 \theta) \delta_{\omega \cdot \omega_{B}}+(1 / 2) S_{t} \sin ^{2} \theta \delta_{\omega, 2 \omega_{B}}\right] .
\end{aligned}
$$

Thus the energy is absorbed from that component of $\mathbf{k}$ which is out of phase with $S_{y}$ and which has the same sense of circular polarization as the magnetic-dipole spin precession. It is readily apparent, however, that $\langle\partial \epsilon / \partial t\rangle_{\mathrm{AR}}$ differs radically from both magnetic spin resonance and quadrupole spin resonance in its angular variation and, even more strikingly, in the possibility of a resonance at $\omega=2 \omega_{B}$. The latter features are characteristic of the electric quadrupole excitation and can be understood as a parametric resonance effect since the ac electric field gradient leads to a periodic modulation of the precession frequency at $2 \omega_{B}$. We now consider several resonance phenomena which occur in the presence of coupling between two different spin systems.

\section{COUPLED SPIN RESONANCE (e.g., HYPERFINE STRUCTURE, SPIN-SPIN SPLITTING)}

Until now, our discussion of spin resonance phenomena has been limited to the case of a single spin system in the presence of a given electromagnetic field which is not affected by the spin system itself. Thus, the results obtained will remain valid for two or more noninteracting spin systems (also in a given electromagnetic field) since the response of the individual spin systems will then take place independently of each other. However, the presence of even weak interactions between the spin systems will fundamentally alter this picture since the individual spin systems will no longer retain their separate identities but instead will respond collectively as a single coupled spin system. ${ }^{27}$ Classically, this collective response will take the form of a precession of the coupled spin system as a whole which can be driven resonantly in analogy with the other types of spin precessions discussed above. We now illustrate these features more quantitatively by using the average-power method to treat such a coupled spin resonance ${ }^{17}$ for a common scalar type of coupling.

The energy and the equations of motion of two different scalar coupled spins $\mathbf{S}$ and $\mathbf{I}$ in the presence of a magnetic field $\mathbf{B}$ will be given by ${ }^{4-7,10}\left(C_{S I}\right.$ is a constant scalar coupling factor)

$$
\begin{gathered}
\epsilon=-\left(M_{S} B\right)(\hat{S} \cdot \hat{B})-C_{S I}(\hat{S} \cdot \hat{I})-\left(M_{I} B\right)(\hat{I} \cdot \hat{B}), \\
d \mathbf{S} / d t=\left(M_{S} B\right)(\hat{S} \times \hat{B})+\left(C_{S I}(\hat{S} \times \hat{I}) \equiv \omega_{C} \times \mathbf{S},\right. \\
d \mathbf{I} / d t=\left(M_{I} B\right)(\hat{I} \times \hat{B})+C_{S I}(\hat{I} \times \hat{S}) \equiv \omega_{C} \times \mathbf{I},
\end{gathered}
$$

where $\omega_{C}$ represents the common precession frequency of the coupled $\mathbf{S}$ and I spin systems and where for simplicity we have neglected electric quadrupole effects. By applying simultaneously both a large dc magnetic field, $\mathbf{B}_{0}$, and a small ac magnetic field, $\mathbf{b}=\mathbf{b}_{0} \sin (\omega t+\phi)$ [as shown in Fig. 1(d)], ${ }^{19}$ it is possible to drive this coupled spin precession resonantly (coupled spin resonance) which, following the average-power method, can be calculated from the free undamped response of the coupled spins; i.e.,

$$
\begin{aligned}
& S_{x} \simeq S_{t} \sin \left(\omega_{C} t\right), S_{y} \simeq S_{t} \cos \left(\omega_{C} t\right), \\
& S_{z}, S_{t} \simeq \text { const },
\end{aligned}
$$




$$
I_{x} \simeq I_{t} \sin \left(\omega_{C} t\right), \quad I_{y} \simeq I_{t} \cos \left(\omega_{C} t\right), \quad I_{z}, I_{t} \simeq \text { const }
$$

Here, $S_{t}$ and $I_{t}$ are the components of $\mathbf{S}$ and $\mathbf{I}$, respectively, which are perpendicular to $\mathbf{B}_{0}$ (i.e., $S^{2}=S_{t}{ }^{2}+S_{z}{ }^{2}$, $I^{2}=I_{t}{ }^{2}+I_{z}{ }^{2}$ ). The values of $\omega_{C}$ for which the coupled coplaner ${ }^{28}$ spin precession described by Eqs. (17) is possible can be found by substituting Eqs. (17) into Eqs. (16). This leads to a quadratic eigenvalue equation for $\omega_{C}$ :

$$
\begin{aligned}
\frac{I_{t}}{S_{t}} \simeq \frac{\left[\omega_{C}-\left(M_{S} / S\right) B_{0}-\left(C_{S I} / S\right)\left(I_{z} / I\right)\right]}{-\left(C_{S I} / I\right)\left(S_{z} / S\right)} \\
\simeq \frac{-\left(C_{S I} / S\right)\left(I_{z} / I\right)}{\left[\omega_{C}-\left(M_{I} / I\right) B_{0}-\left(C_{S I} / I\right)\left(S_{z} / S\right)\right]}
\end{aligned}
$$

The power absorbed by the coupled $\mathbf{S}$ and $\mathbf{I}$ spin systems follows from Eqs. (1), (15), and (16) ${ }^{19,20}$ as

$$
\begin{array}{r}
\partial \epsilon / \partial t \simeq-\left(M_{S} \mathbf{S}+M_{I} \mathbf{I}\right) \cdot d \mathbf{b} / d t, \\
\langle\partial \epsilon / \partial t\rangle_{\mathrm{CR}} \simeq-\left[(1 / 2)\left(M_{S} / S\right) S_{t}+\left(M_{J} / I\right) I_{t}\right] \\
\times b_{0} \omega \sin \theta \cos \phi \delta_{\omega_{1} \omega_{C}}
\end{array}
$$

It is often convenient to write the two different values of $\omega_{C}$ defining the resonant coupled spin response in Eq. (20) as $\omega_{\mathrm{CS}}$ and $\omega_{\mathrm{Cl}}$ for those eigenfrequencies which approach the precession frequencies of the individual $\mathbf{S}$ and $\mathbf{I}$ spin systems, respectively, in the weak-coupling limit, $C_{S I} \ll$ $M_{S} B_{0}, M_{l} B_{0}$; i.e.,

$$
\begin{aligned}
\omega_{C}=\omega_{\mathrm{CS}}\left(C_{S I} \ll\right. & \left.M_{S} B_{0}\right) \\
& \simeq\left(M_{S} / S\right) B_{0}+\left(C_{S I} / S\right)\left(I_{z} / S\right), \\
\omega_{C}=\omega_{C l}\left(C_{S I} \ll\right. & \left.M_{I} B_{0}\right) \\
& \simeq\left(M_{I} / I\right) B_{0}+\left(C_{S I} / I\right)\left(S_{z} / S\right) .
\end{aligned}
$$

The coplanar precession of both spin systems at the angular frequency $\omega_{C S}$ given by Eq. (21a) is the classical picture of hyperfine structure observed in many ESR experiments. In such cases, $\mathbf{S}$ represents an electron spin while I represents an interacting nuclear spin which slightly "shifts" the noninteracting precession frequency of the electron. The interaction itself results from the direct contact of the ( $S$-state) electron with the nucleus in question. ${ }^{1-3,5,6}$

In similar fashion, Eqs. (21a) and (21b) taken together represent the classical picture of spin-spin splitting in NMR experiments where $\mathbf{S}$ and I are different nuclear spins which precess together at frequencies slightly different from the precession frequencies of the $\mathbf{S}$ and I nuclear spin systems in the absence of a coupling interaction. The actual coupling between the two nuclear spins takes place indirectly due to their mutual polarization of an intervening electron cloud. $2,3,5,6$

In general, the coupling interaction between the two spin systems cannot be assumed to be weak, and the exact values of $\omega_{C}$ (and, hence, of $\langle\partial \epsilon / \partial t\rangle_{\mathrm{CR}} \sim \omega_{C}$ ) from Eq. (20) will differ substantially from the precession frequencies of the individual spin systems in the absence of coupling. Such a situation would apply to strong exchange coupling as well as to other cases of strongly coupled spin systems described by different coupling interactions, e.g., chemical exchange $^{3,6}$ and ferromagnetic (or antiferromagnetic ${ }^{1,7,10}$ ) resonance. In addition, more general coupling interactions involving three or more interacting spin systems or coupling between the spin and spatial degrees of freedom (e.g., spin-orbit coupling ${ }^{29}$ ) will also lead to coupled spin re- sponses qualitatively different from the weak-coupling limit. In such cases, the average-power method is especially advantageous since it allows the calculation of the resonant spin response to be reduced to a straightforward solution of the eigenvalue equation for the free undamped precession frequencies of the coupled spin system. Such a procedure is, of course, the classical counterpart of the diagonalization procedure used to find the energy level spectrum in quantum-mechanical calculations. ${ }^{30}$ In practice, however, the classical calculation is usually much simpler since the number of precession eigenfrequencies (each of which corresponds directly to a possible spin resonance frequency of the coupled spin system) is always less than the total number of energy eigenvalues appearing in the analogous quantum-mechanical calculation. This leads to an additional advantage, viz., the existence and values of the spin resonance frequencies appear naturally without the explicit introduction of quantum-mechanical selection rules. ${ }^{31}$ The effects of quantization on this classical picture-where they ae important - can then be taken into account a posteriori by quantizing the spin resonance frequency, $\omega_{\text {res }}$, itself. This procedure as well as several other useful extensions of the average-power method will now be discussed briefly.

\section{DISCUSSION}

One immediate extension of Eq. (1) would be to apply the Bohr-Sommerfeld quantization ${ }^{32}$ rules to the results already derived by substituting $m \hbar$ and $(n+1 / 2) h$ for $S_{z}$ (or $I_{z}$ ) and $S$ (or $I$ ), respectively, where $m$ and $n$ are integral multiples of $1 / 2$. The resulting quasiclassical approximations for $\langle\partial \epsilon / \partial t\rangle$ and $\omega_{\text {res }}$ differ by less than $10 \%$ from corresponding quantum-mechanical values for small $m$ and $n$, and are indistinguishable from the exact result for large $m$ and $n$. This agreement can be improved still further by suitably generalizing the Bohr-Sommerfeld quantization rules $^{33}$ and is, in fact, always exact (for $\omega_{\text {res }}$ ) in cases where the dominant spin response is linear in the spin magnetization $\mathbf{S}$ (and I), e.g., the magnetic-dipole and coupled spin precessions discussed above.

Another extension would be to consider the effects of damping due to the spin environment on the various spin resonance phenomena. Such effects can be taken into account very simply in the average-power method by using the damped time-dependent value of the transverse spin magnetization with the averaging time, $T$, in Eq. (1) chosen such that $2 \pi / \omega \ll T \ll T_{2}$, where $\omega$ is the angular frequency of the ac driving field (or field gradient) and $T_{2}$ is a transverse relaxation time characterizing the damping. $5-7$ Thus, for example, the damped time dependence of the transverse spin magnetization could be written either as $S_{t}$ $\sim \exp \left(-t / T_{2}\right)$ or $S_{t} \sim \exp \left[-(\pi / 4)\left(t / T_{2}\right)^{2}\right]$. respectively, to describe damping which is primarily due to fast or slow variations in the local ac electromagnetic fields. ${ }^{7}$ The former would result in a so-called Lorentzian resonance line shape for $\langle\partial \epsilon / \partial t\rangle_{\text {res }}$ as a function of $\omega-\omega_{\text {res }}$, whereas the latter would give rise to a so-called Gaussian resonance line shape. ${ }^{11,34}$ In general, however, the resonance line shape will be influenced by factors other than damping alone ${ }^{5-10}$ (e.g., by variations in the local dc electromagnetic fields) and, in the absence of a detailed description of the actual broadening mechanisms involved in a particular case, the most valid approach is to simply replace the Kronecker delta, $\delta_{\omega, \omega_{\text {res }}}$, appearing in $\langle\partial \epsilon / \partial t\rangle_{\text {res }}$ by a resonance line 
shape factor $g\left(\omega-\omega_{\text {res }}\right)$ in analogy with the quantummechanical case. ${ }^{11,35}$

Another important extension would be to include explicitly the effect of the ac driving field (or field gradient) on the spin precessions. Such effects have been neglected above but can lead to significant changes in the longitudinal spin magnetization $S_{t}$ (and hence in $\langle\partial t / \partial t\rangle_{\text {res }} \sim S_{t}$ since $S_{t}{ }^{2}=S^{2}-S_{z}{ }^{2}$ ) even when the amplitude of the ac driving field (or field gradient) is less than the spin resonance linewidth. ${ }^{35}$ In contrast to the case of damping, it is possible to give a generally valid description of changes in the longitudinal spin magnetization in terms of a single longitudinal relaxation time, $T_{1}$, which characterizes the approach of $S_{z}$ to some thermal equilibrium value. Thus, even a simple expression for longitudinal spin relaxation such as $d S_{z} / d t=\left(S-S_{z}\right) / T_{1}$ permits a wide range of phenomena, e.g., saturation, to be treated quantitatively. For example, the effects of such a longitudinal spin relaxation term will appear naturally in the average-power calculations as a steady-state ${ }^{36}$ value of $S_{z}$ that is dependent on the magnitude of the ac driving field (or field gradient). This dependence of $S_{z}$ on the ac driving field (or field gradient) - the so-called saturation phenomenon-is, of course, the basis of many double spin resonance ${ }^{37}$ phenomena in which the excitation of a given type of spin resonance is detected indirectly by the application of a second ac field (or field gradient) in order to saturate the original spin resonance. Thus, the inclusion of two longitudinal relaxation times $T_{I S}$ and $T_{1 I}$ (and a third cross-relaxation time $T_{\mid S I}$ ) for the scalar coupled electron (S)-nuclear (I) spin system considered above would allow saturation coupled spin resonance (so-called ENDOR) phenomena to be calculated. In such cases, the calculation in terms of the average power not only provides a convenient method of calculating the resonant response but also gives a very helpful physical insight into the nature of complicated spin resonance phenomena that is difficult to obtain from the standard treatment of such phenomena.

'J. Blakemore, in Solid State Physics (Saunders, Philadelphia, 1974), Chap. 5; R. L. Armstrong and J. D. King, in The Electromagnetic Interaction (Prentice-Hall, London, 1973), Chap. 16.

${ }^{2} \mathrm{C}$. Kittel, in Introduction to Solid State Physics (Wiley, London, 1966), Chap. 16; A. H. Morrish, The Principles of Magnetism (Wiley, London, 1966).

${ }^{3} \wedge$. Carrington and A. D. McLachlan, Introduction to Magnetic Resonance (Harper and Row, London, 1967); J. E. Wertz and J. R. Bolton, Electron Spin Resonance (McGraw-Hill, Düsseldorf, 1972).

${ }^{4} \mathrm{C}$. P. Slichter, Principles of Magnetic Resonance (Harper and Row, London, 1963); T. R. Schumacher, Introduction to Magnetic Resonance (Benjamin, Amsterdam, 1970).

${ }^{5}$ Because of these difficulties, the case of an arbitrarily oriented, linearly polarized ac driving field, corresponding to many experimental situations, is not treated analytically in elementary textbooks. More important, however, is the fact that the Bloch equations are known to be invalid in many important cases, e.g., in solids, or in small or inhomogeneous extremal fields. In fact, calculations based on the Bloch equations are not even qualitatively correct in the presence of inhomogeneous fields: A. Abragam, The Principles of Nuclear Magnetism (Oxford U.P., London, 1970); A. Abragam and B. Bleaney, Electron Paramagnetic Resonance of Transition Ions (Clarendon, Oxford, 1970).

${ }^{6} \mathrm{G}$. E. Pake and T. L. Estle, The Physical Principles of Electron Paramagnetic Resonance (Benjamin, Amsterdam, 1973); C. Poole and H. Farach, The Theory of Magnetic Resonance (Wiley, London, 1972).
${ }^{7} \mathrm{~J}$. Talpe, The Theory of Experiments in Paramagnetic Resonance (Pergamon, Braunschweig, 1971); M. Goldman, Spin Temperature and Nuclear Magnetic Resonance in Solids (Oxford U.P., London, 1970); W. Th. Wenckebach et al., Phys. Rep. 14, 181 (1974).

${ }^{8}$ H. G. Dehmelt, Am. J. Phys. 22, 110 (1954); 22, 317 (1954); T. P. Das and E. L. Hahn, in Nuclear Quadrupole Resonance Spectroscopy: Solid State Physics, Suppl. I (Academic, London, 1958); E. A. C. Lücken, Nuclear Quadrupole Coupling Constants (Academic, London, 1969).

${ }^{9}$ D. Bolef, Science 136, 359 (1962); S. A. Al'tshuler et al., Sov. Phys. Usp. 4, 880 (1962); D. I. Bolef, in Physical Acoustics, Vol. IV, Part A, edited by W. P. Mason (Academic, London, 1966), Chap. 3.

${ }^{10} \mathrm{~S}$. Foner, in Magnetism, Vol. I, edited by G. T. Rado and H. Suhl (Academic, London, 1966), Chap. 9; V. A. Atsarkin and M. I. Rodak, Sov. Phys. Usp. 15, 251 (1972); E. Turov and M. Petrov, Nuclear Magnetic Resonance in Ferro- and Antiferromagnets (Halsted, New York, 1973).

I'The method, for $T \rightarrow \infty$, has been briefly mentioned before but, as far as we know, has not been applied to the spin resonance phenomena under discussion here. J. Winter, Magnetic Resonance in Metals (Oxford U.P., London, 1971), p. 3. It is worth noting that the mathematical averaging process described by Eq. (1) often corresponds to the experimental situation since many measuring devices do, in fact, record the signal averaged over a large number of periods of the electromagnetic field used to excite the spin resonance. It is also worthwhile pointing out that the method is not restricted to the case of spin resonance and a large number of other resonance phenomena could be treated profitably by the same technique.

${ }^{12}$ Such a power series expansion of $\partial \epsilon / \partial t$ in the ac driving electromagnetic field is, of course, always possible as long as the effect of the driving field on $\partial f / \partial t$ is small. This is always satisfied arbitrarily close to the resonance condition $\omega=\omega_{\text {res }}$ as long as the magnitude of the ac electromagnetic field (in frequency units) is small in comparison with $\mid \omega-$ $\omega_{\text {res }} \mid$; i.e., $\omega_{\text {ac }} \ll\left|\omega-\omega_{\text {res }}\right|$. Physically, this expresses the fact that the resonance condition cannot be determined to better than $\pm \omega_{\text {ac }}$ by using the standard power series method for a free undamped spin system. It is worth noting that the same situation obtains in the quantum-mechanical case. K. Gottfried, Quantum Mechanics, Vol. I (Benjamin, Amsterdam, 1966), Chap. 9. The mathematical degeneracy or indeterminacy of the resonance condition can be removed by more advanced perturbation techniques beyond the scope of an introductory treatment: sec, for example, D. Montgomery and T. W. Ruijgrok, Am. J. Phys. 33, 946 (1965); L. D. Landau and I. M. Lifshits, Quantum Mechanics: Non-Relativistic Theory (Pergamon, London, 1965).

${ }^{13}$ Both the quantum-mechanical approach and the usual classical calculation in terms of the spin magnetization apply a similar first-order expansion of the spin response in the driving electromagnetic field.

${ }^{14} \mathrm{Spin}$ relaxation effects can be treated by a simple extension of the average-power method as presented in the discussion above. The average-power method provides a simpler, more generally valid calculation of such effects than the conventional calculation of $d \mathbf{S} / d t$ using the Bloch equations. This advantage results from the fact that $\partial \epsilon / \partial t$ is linear in both the ac driving electromagnetic field and the spin magnetization $\mathbf{S}$. Thus, spin relaxation effects such as damping or spin lattice relaxation can be treated by considering correspondingly lower order terms of a power series expansion of $\mathbf{S}$ in the ac driving electromagnetic field.

${ }^{15}$ More rigorously, the equation of motion for the quantum-mechanical expectation value of the spin magnetization vector $\langle\mathbf{S}\rangle_{\mathrm{QM}}$ represents a precession of $\langle\mathbf{S}\rangle_{\mathrm{QM}}$ about the directions of an external electromagnetic field and its spatial derivatives.

${ }^{16}$ The term magnetic-dipole spin precession results from the fact that the magnetic-dipole vector and the spin magnetization vector are collinear.

${ }^{17}$ The terms used to describe the various spin resonance phenomena under discussion here are defined in the text and in Fig. 1.

${ }^{18}$ The use of a caret ( $)$ and boldface type have the usual meanings of a unit vector and an ordinary vector, respectively; e.g., $\mathbf{S} \equiv S \hat{S}$, where $S$ is the magnitude of $\mathbf{S}$.

${ }^{19}$ The choice of the geometry shown in Fig. 1 as well as the phase facter, $\phi$, leads to no loss of generality since both the orientation of the $y$ axis and the initial instant, $t=0$, are arbitrary. 
${ }^{20}$ The appearance of the factors $M_{S}$ and $\mathbf{b}$ illustrates the so-called magnetic-dipole nature of the excitation.

${ }^{2} \mathrm{E}$. Ley-Koo, Am. J. Phys. 40, 516 (1972). The electric quadrupole moment as given here is defined as $Q_{S} \equiv(1 / 2) Q^{\prime}(n+1) /(2 n-1)$, where $Q^{\prime}=\int \rho(\mathbf{r}) d V\left(3 z^{2}-r^{2}\right)$ is the standard expression for the quadrupole moment and $n$ is an integral multiple of $1 / 2$ with $S=n \hbar$. (The electric quadrupole tensor is assumed to be axially symmetric about the spin direction.)

${ }^{22}$ The electric field gradient is assumed to be axially symmetric for simplicity. The vector $\mathbf{K}$ thus represents a vector whose magnitude is equal to the value of the electric field gradient tensor along the axial symmetry direction (given by $\mathbf{K})$; i.e., $\mathbf{K} \equiv(\nabla \mathbf{E} \cdot \hat{K})$, where $\mathbf{E}$ is the electric field vector.

${ }^{23}$ Equation (8) represents the two leading spin-dependent terms of the multipole expansion of the energy of a spin charge system in the presence of an external electromagnetic field. S. De Benedetti, Nuclear Interactions (Wiley, London, 1964).

${ }^{24} \mathrm{Spin}$ resonance phenomena are also possible in cases where the spin precessions are comparable. V. S. Grechiskin and N. E. Ainbinder, Sov. Phys. Usp. 6, 566 (1964). Such spin resonance phenomena could be treated using the average-power method although the calculations will, of course, be more complicated.

${ }^{25}$ The presence of the factors $Q_{S}$ and $k$ illustrates the so-called electric quadrupole nature of the excitation.

${ }^{26} \mathrm{An}$ approximate expression for $\partial \epsilon / \partial t$ can be obtained by neglecting the electric quadrupole term in Eq. (8) in comparison with the magnetic dipole term, viz. $\partial \epsilon / \partial t \sim-\left(M_{S} B\right)(\mathbf{B} \cdot d \mathbf{S} / d t)$. The corresponding approximate value of $\langle\partial \epsilon / \partial t\rangle$ will differ from the exact expression [Eq. (13)] by the factor $\omega / \omega_{\text {res. }}$. Similar approximations could also be made for the other spin resonance phenomena.

${ }^{27}$ This qualitative difference between the case of no coupling and the case of weak coupling is important in the classical interpretation of hyperfine structure, spin-spin splitting, and double resonance phenomena.

${ }^{28}$ The projections of $\mathbf{S}$ and $\mathbf{I}$ on to the $x-y$ plane are collinear.

${ }^{29}$ In such cases the effect of the coupling interaction is usually much less on one (or more) of the interacting spin systems than on the others, and it is sometimes convenient to treat the motion of this spin system as if it were independent of the other spin systems. Thus, spin-orbit coupling can be calculated assuming a given orbital motion of the spin. Similarly, the chemical shift could be treated assuming a given magnetic fielddependent (diamagnetic) motion of the electrons in the vicinity of a nucleus.

${ }^{30} \mathrm{It}$ is often convenient to find approximate solutions to the eigenvalue equation for the coupled spin precession frequencies by projecting the different spin precessions along a common direction. This would correspond to the quantization of different terms in the Hamiltonian along a given direction in the quantum-mechanical approach.

${ }^{31}$ This advantage is especially well pronounced for spin resonance phenomena which are usually treated as second-order or forbidden (electric quadrupole) transitions in the quantum-mechanical approach.

${ }^{32}$ Y. Beers, Am. J. Phys. 40, 1139 (1972).

${ }^{33}$ R. Klingbeil, Am. J. Phys. 41, 297 (1973).

${ }^{34}$ Such a calculation readily demonstrates the absorption and dispersion line shapes as well as the different contributions of the two circularly polarized components of the ac driving field (or field gradient) to the $\langle\partial t / \partial t\rangle_{\text {res }}$ resonance curve. It is important to note that the Gaussian line shape can also result from nondamping effects such as variations in the local dc field. G. W. Parker, Am. J. Phys. 38, 1432 (1970); A. M. Stoneham, Rev. Mod. Phys. 41, 82 (1969).

${ }^{35}$ The power series expansion of $\partial \epsilon / \partial t$ in the ac driving field (or field. gradients) is no longer valid when the magnitude of this field (or field gradient) is comparable to or exceeds the resonance linewidth. In practice, longitudinal (spin lattice) relaxation effects begin to play a dominant role before this can occur.

${ }^{36}$ The steady-state condition could, for example, be defined as $\langle d \mathbf{S} / d t\rangle$ $=0$, with the averaging carried out as in Eq. (1).

${ }^{37}$ One example of such a technique is the acoustic saturation of a magnetic spin resonance signal. D. Bolef, Ref. 9. Likewise, the application of ac magnetic fields at each of the two precession eigenfrequencies of a coupled electron-nucleus spin system is a technique frequently employed to detect indirectly the excitation of the coupled spin system at one precession eigenfrequency by its effect on the precession of the coupled spin system at the other eigenfrequency (the ENDOR technique). A. Abragam and B. Bleaney, Ref. 5. 